\title{
A Contradiction-Based Approach for Innovative Product Design
}

\author{
Yao-Tsung Ko, Chia-Chen Lu and Li-Hui Lee \\ Department of Industrial Design, Tunghai University, Taiwan \\ No.1727, Sec. 4., Taiwan Boulevard, Xitun District, Taichung City, 40704
}

\begin{abstract}
Without creativity in design there is no potential for innovation. This paper investigates the role of contradictions in enhancing creativity in product design. Based on the inventive principles of TRIZ, this paper presents a novel design method by integrating technical and physical contradiction analysis methods into the conceptual design activities of new product development (NPD). Despite the recognized importance of innovative design, there is a lack of the systematic and effective design-thinking process that can covers all conceptual design activities. To address this gap, a sharper and fundamental model of the problem-solving is created for innovative product design based on the contradiction-oriented concept. Eventually, one case study is employed to illustrate the method and the result validates that it can help designers produce more creative outcomes in product design.
\end{abstract}

\section{Introduction}

When studying the complexity and numbers of constraints and contradictions in product design projects, to the observer it sometimes seems miraculous how designers can work in such environments-let alone produce outstanding creative outcomes. The importance of conflicts and constraints in design is a well-studied subject [1], but the relationship between contradictions and creativity seems to be fairly unexplored. It not only within design but also in the creativity literature in general. Sternberg and Kaufman [2] point out the contradictions exist on creativity in their recent research. The engineering design literature seems to pay less attention to it, despite engineering design being recognised as a constraint-based domain [3], [4] where creativity plays an important role. The term "constraints" often implies the restrictions of free and creativity [5], but numerous researchers pointed out that without conflicts and constraints there can be no creativity [6], [7]. Not only can contradictions enhance creativity, they are key elements for all creative activities [1], [2]. Some types of contradictions can be highly limiting for creativity [8], but without contradictions and constraints there is no problem to resolve and no potential for creativity. Regarding contradictions as important drivers for creative design, it becomes important and interesting to study how the contradictions in design process affect creativity. Seeking to explore this relationship in product design, this paper proposed a creative design-thinking approach based on technical and physical contradictions concept. This study focused on three main areas: (1) how can contradictions be created and managed to give the best possible room for innovation, (2) how do designers think with contradictions as part of their creative process and (3) the creative role of contradictions and their abstraction level. Creativity and contradictions are inevitably related. To understand this complex relationship is not only important for realizing creativity in product design, it is also essential to support real-world design activities. Finally, work within this topic can contribute explicitly to understanding and supporting creativity in product design.

\section{Methodology}

\subsection{Technical contradiction}

The core concept of TRIZ is engineering contradiction. An engineering contradiction is a situation, in which an attempt to improve one parameter of an engineering system leads to the worsening of another parameter. Engineering contradiction also known as "Technical Contradiction". Altshuller's Matrix is a problem-solving tool that recommends inventive principles for solving engineering contradictions. What are Altshuller's findings? He studied the engineering problems and their resolution by analyzing thousands of patent documents in Russia. The followings are his key findings. He identified that 39 typical parameters are involved in most engineering contradictions. For example, length of a stationary object, weight of a stationary object etc. Typical solutions to typical contradictions seem to work successfully in most situations. He generalized these solutions and called them "Inventive Principles". There are 40 Inventive Principles in TRIZ [9]. 


\subsubsection{Altshuller's matrix}

Only the engineering contradiction cannot help designers create novel ideas. The users must employ the Altshuller's Matrix to identify the improve and worsen parameters then produce the advised inventive principles. The Altshuller's Matrix also known as "TRIZ Matrix". How to use the TRIZ Matrix? The first step is to transform a specific technical contradiction to a typical one. It is critical to formulate a typical contradiction using the 39 universal technical parameters from TRIZ Matrix. Next, we identify and apply recommended principles from the matrix. The result is a set of general recommendations for resolving the identified contradiction. Finally, those general recommendations must be translated into specific technical ideas that can solve the initial technical contradiction.

\subsubsection{Process of resolving technical contradictions}

Inventive problems are written in the form of "If-thenbut". The procedure of resolving technical contradictions are listed as below.

(1) Identifying specific parameters.

(2) Identify the parameters in the engineering contradiction.

(3) Identifying typical parameters.

(4) Identify from Altshuller's list those Typical Parameters that are similar in meaning to the specific parameters or are derivatives of specific parameters.

\subsection{Physical contradiction}

A physical contradiction is two opposite requirements placed upon a single physical parameter of an object. These requirements are caused by the conflicting requirements of an engineering or technical contradiction. There is Moe power and uniqueness in physical contradiction analysis. The engineering contradictions are formulated for technical parameters of the engineering system. Physical contradictions represent the next step of abstraction. Physical contradictions are formulated for physical parameters which can be resolved in Time, Space, Affiliation, and System Hierarchy. This allows TRIZ Inventive Principles to be used more effectively. Physical contradictions present a sharper and fundamental model of the problem-solving than the traditional TRIZ engineering contradictions, thereby enabling more powerful solutions.

The steps of physical contradiction analysis are presented as follows:

(1) Formulate two justified contradictory (opposite) requirements for one of the parameters of the engineering system or its components.

(2) Determine what typical approaches are applicable to the physical contradiction at hand.

(3) Identify the inventive principle needed relevant to the chosen approach. Applying inventive principles leads to ideas that solve the initial physical contradiction.

A physical contradiction is a situation in which an engineering system demands contradictory values from the same parameter. For example, to improve the quality of nail penetration, the contradiction is that the hammer should be made heavy, but to improve its handling it should be made light. The design-thinking process will be shown as follows:

To (achieve goal 1) (the subject parameter) should be $(+\mathrm{A})$; But

To (achieve goal 2) (the subject parameter) should be $(-\mathrm{A})$

In order to formulate the requirement of the system we must indicate the definitions including:

(1) The demanded parameter

(2) Goal 1 and Requirement 1; Goal 2 and Requirement 2

There are three categories of ways to solve physical contradictions:

(1) Separating contradictory demands

(2) Satisfying contradictory demands

(3) Bypassing contradictory demands (System transition)

\subsubsection{Separating contradictory demands}

Five methods of separation in the separating contradictory demands are proposed as below.

(1) Separation in Space

(2) Separation in Time

(3) Separation in Relation

(4) Separation in System Levels

(5) Separation between Parameters

Separation removes the contradiction and enables each demand to be met. The designers can get the initial solutions for design problems using these five separation methods.

\subsubsection{Separation in space}

If two contradictory demands are required at different locations within an engineering system, separate the contradictory demands. The keyword of design-thinking is "where". Ask where do the system want $+\mathrm{A}$ ? and where do the system want $-\mathrm{A}$ ? If the answer can be different, we can separate the opposite demands by space. Resolving the physical contradiction using "Separation in Space", there are six Inventive Principles of TRIZ recommended for separation concepts in space:
\#1-Segmentation
\#2-Separation (taking out)
\#3-Local quality
\#7-Nested doll
\#4-Symmetry change (asymmetry)
\#17-Dimensionality change (another dimension)

\subsubsection{Separation in time}

If the contradictory demands are required at different times, separate them using "Separation in Time" method. For example, how to resolve the design problem of the eye of the needle. The contradiction is that the eye of the needle shoule be large for directing the thread easily into it, but the eye of the needle should be small to avoid 
damage to the clothes. The keyword of design-thinking is "when". Ask when do I want $+A$ ? and when do I want $-A$ ? The separation method in time can be identified as follows:

(1) Separation in Space cannot be applied

Location of contradictory demands is same i.e., the hole of the needle

(2) Separation in Time can be applied

"Hole should be large" is required before sewing

"Hole should be small" is required while sewing

Since the contradiction demands are for different times, this physical contradiction can be separated using "Separation in Time". Five Inventive Principles are recommended for resolving the physical contradiction using "Separation in Time":

\#15-Dynamization

\#34-Discarding and recovering

\#10-Preliminary action

\#9-Preliminary counteraction

\#11-Prior compensation

\subsubsection{Separation in relation}

If the contradictory demands are required for different components of the supersystem, separate them using 'Separation in Relation' method. For instance, how to design a "Window" with good function. The contradiction is that a window should be open to allow the air to come in but the window should be closed to not allow the sun rays to come in. The window is a system. Sun and air belong to supersystem components. The method of Separation in Relation can be proposed as follows:

(1) Separation in Space cannot be applied

The contradictory demands are required for the same window

(2) Separation in Time cannot be applied

"Allowing air" and "not allowing sunlight" must happen at the same time.

(3) Separation in Relation can be applied

"Window should be open" is required for air

"Window should be closed" is required for sunlight

Sunlight and air are two different components of the supersystem. There are five Inventive Principles proposed for "Separation in Relation".

\#40-Composite material

\#31-Porous materials

\#32-Optical properties changes

\#3-Local quality

\#19-Periodic action

\#17-Dimensionality change

\subsubsection{Separation in system level}

If one of the contradictory demands is required at the Subsystem or Supersystem level, separate them using "Separation in System Level" method. Separate the contradictory demands in separate system levels. For example, how to design a "Strong Rope". The engineering problem is to produce a rope which can meet the following requirements:

(1) As strong as cast iron
(2) Flexible enough to be folded and stored in a small place

There are four Inventive Principles are recommended for resolving the physical contradiction using "Separation in System Level" method:

\#1-Segmentation

\#5-Merging

\#33-Homogeneity

The designer can apply the Inventive Principle \#1Segmentation to resolve the problem of Strong Rope. The Inventive Principle \#1-Segmentation means that:

(1) Divide an object into independent parts

(2) Make an object easy to disassemble

(3) Increase the degree of fragmentation or segmentation

The final design solution is based on the "chain" concept. The components of the chain are made of cast iron and are rigid but the whole chain is flexible and can be folded for storage purpose.

\subsubsection{Separation between parameters}

When $+\mathrm{A}$ and $-\mathrm{A}$ are required for one parameter, check if the parameter is dependent on multiple independent parameters. Let $+\mathrm{A}$ requirement apply on one composing parameter and let $-\mathrm{A}$ requirement apply on another composing parameter. The formulation of separation between parameters is shown as follows:

$\mathrm{Y} \rightarrow$ big; $\mathrm{Y} \rightarrow$ small

Actually $\mathrm{Y} \rightarrow$ big is $\mathrm{X} 1$ to be big or $\mathrm{Y} \rightarrow$ small is $\mathrm{X} 2$ to be small.

$\mathrm{Y}=$ function $(\mathrm{X} 1, \mathrm{X} 2, \ldots)$

Let $\mathrm{X} 1$ and $\mathrm{X} 2$ handle the opposing requirements separately.

\subsection{Converting technical contradictions to physical contradictions}

When two parameters are functions of same parameter, a technical contradiction can be converted into a physical contradiction. That means we located a core fundamental parameter. In general, converting from a technical contradiction to a physical contradiction makes the case more inventive as we are solving a more controlling and core problem. In that case, the solution are usually "stronger". The relationships between technical contradiction and physical contradiction is presented as Fig. 1.

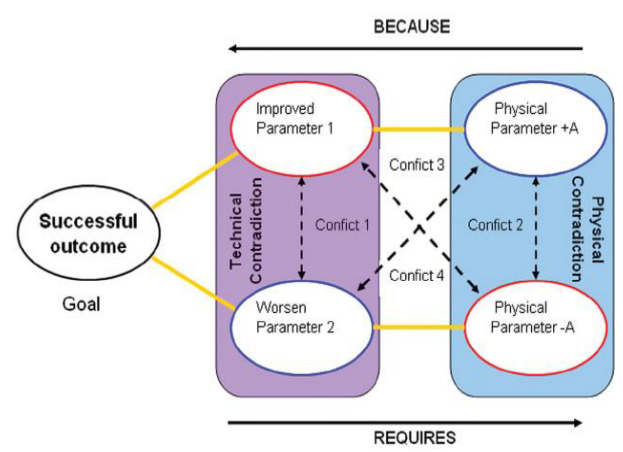

Figure 1. Identify a technical contradiction and corresponding physical contradiction. 


\subsubsection{Four paths of resolving design problems}

In Fig. 1, the design problems can be resolved by the two ways of considering technical contradiction and physical contradiction. There are four paths of resolving design conflicts between technical and physical contradiction as shown follows.

(1) Resolve technical contradiction (conflict 1) between improved and worsen parameters (parameter 1 and 2).

(2) Resolve physical contradiction (conflict 2) between physical parameter $+\mathrm{A}$ and $-\mathrm{A}$.

(3) Resolve technical contradiction and physical contradiction (conflict 3) between improved parameter and physical parameter $+\mathrm{A}$.

(4) Resolve technical contradiction and physical contradiction (conflict 4) between worsen parameter and physical parameter -A.

The contradiction matrix and inventive principles of TRIZ can be used to resolve conflict 1,3 and 4 . The related algorithm for resolving physical contradiction mentioned above can be used to resolve conflict 2 .

\section{A case study}

The creating process of a innovative screwdriver is presented as an example in this case study. The technical and physical contradictions are adopted to resolving the screwdriver design problems for creatiing product innovation at the same time. The main function of the screwdriver is to turn or release screws. It will raise insufficient torque when driving bigger screws. Meanwhile the screwdriver is not good in the driving efficiency when driving longer screws. The innovative goal of designing screwdriver is to improve the torque and efficiency in operation.

\subsection{Identify the contradiction of the system}

According to the goal of design screwdriver, the contradiction of the system can be obtained. The contradiction is when we drive a screw with high rotation speed, it will raise a low operation toeque. These two conflict parameters are "operation efficiency" and "operation torque". The improving technical parameter is "\#9-Speed" and the worsen technical parameter is "\#10Force".

\subsection{Analyze the design problem and conflict}

After identifying the contradictions the conflict matrix is used to analyze the design problem and solve the technical contradictions. The related inventive principles is obtained from the contradiction matrix. Based on the 39 technical parameters and 40 inventive principles of TRIZ, the design solution trigger and specific solution can be obtained as follows:

\#13-The other way around

\#15-Dynamization

\#19-Periodic

\#28-Mechanics substitution

\subsection{Convert technical contradiction to physical contradiction}

In order to produce more creative outcomes we must convert technical contradiction to physical contradiction for more shaper and advanced design solutions. There are two conflict technical parameters in the design problem. When the screwdriver drives the longer screw, it need higher rotation speed to add operation efficiency. When the screwdriver drives the bigger screw, it need lower rotation speed to add operation torque. The physical contradiction is a situation in which the screwdriver design demands the contradictory values from the same parameter "Rotation Speed". Therefore, the technical contradiction can be converted to the physical contradiction as shown as following Fig. 2.

\subsubsection{Use separation principles}

The physical contradiction parameter "rotation speed" can be produced from the analysis of the above conevrting process. The condition meets the following design-thinking semantics.

To improve the operation efficiency Requires higher rotation speed; BUT

To improve the operation torque Requires lower rotation_speed.

\subsubsection{Transition to a different system}

The transtion principles can be also adopted to resolve the design problems unless the separation principles. The design thinking semantics can be shown as follows.

+ A: high rotation speed; - A: low rotation speed

Can high rotation speed $(+\mathrm{A})$ and low rotation speed (-A) be transferred to another system? The answer is positive. To establish a new gear module system which can offer different rotation speed can resolve this design problem.

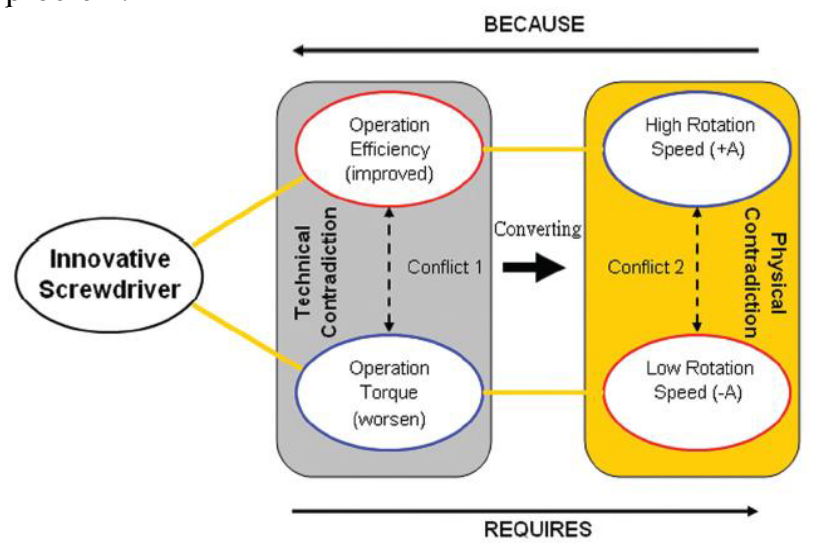

Figure 2. The physical contradiction parameter converting of innovative screwdriver.

\subsection{Visualize the design concept}

Based on the analysis of technical and physical contradiction, the concrete design concept for innovative driver can be presented as Fig. 3 . 


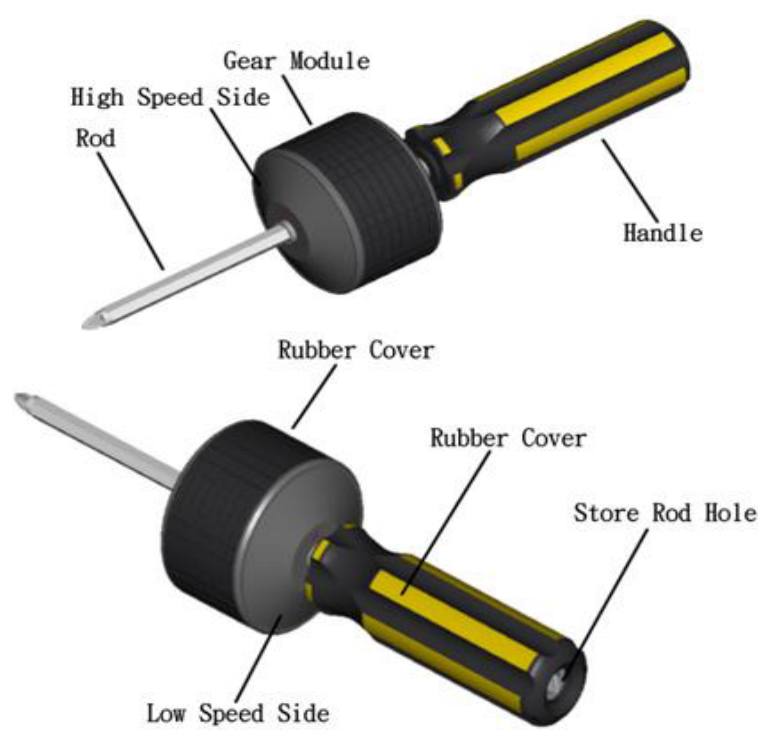

Figure 3. The final innovative driver design.

\section{Conclusions}

Various methods focus on increasing creativity in conceptual design. Which methods are capable of producing more creative outcomes is an important practical problem. Creativity is an integral and essential part in the product design process. Without creativity in design there is no potential for innovation, which is where creative ideas are actually implemented and transformed into commercial value. Also, without contradiction in design there is no novel ideas for creativity. As the contradictions were used both to assess and to explain creativity, it shows that in complex designs, creativity is closely linked to the contradictions used to reach the result. Considering this crucial creative role of contradictions, the reviewpoint of this paper is not considering contradictions as fixed single entities that can each be understood and researched in isolation, but as complex, dynamic and interdependent sets of contradictions. Based on the TRIZ concept, this paper presents a novel design-thinking approach by integrating technical and physical contradiction analysis methods into the conceptual design activities of New Product Development (NPD). The proposed design problemsolving method really can help designers create novel ideas for new product design by the verification of the case study. Meanwhile, by employing a multidimensional understanding through exploring contradictions beyond binary typologies and singular dimensions, creativity research can offer a more realistic and relevant starting point for both researchers and practitioners seeking to stimulate creativity through contradictions.

\section{References}

1. M. Darlington, Cognition and the engineering design requirement, Thesis (PhD), University of Bath, (2002)

2. R.J. Sternberg and J.C. Kaufman, Constraints on creativity, Cambridge handbook of creativity. New York: Cambridge University Press, 467-482, (2010).

3. S. Ajit, Constraint capture and maintenance in engineering design, AI EDAM, 22 (4): 325-343 (2008)

4. M. Stacey and C. Eckert, Reshaping the box: Creative designing as constraint management, International Journal of Product Development, 11 (34): 241-255 (2010).

5. P.D. Stokes, Creativity from constraints: What can we learn from Motherwell? From Modrian? From Klee? The Journal of Creative Behavior, 42 (4): 223 236 (2008).

6. P.D. Stokes, Creativity from constraints, New York: Springer, (2005)

7. J.H. Dyer, H.B. Gregersen and C.M. Christensen, The innovator's DNA. Harvard Business Review, 87 (12): 60-67 (2009).

8. A. Salter and D. Gann, Sources of ideas for innovation in engineering design, Research Policy, 32 (8): 1309-1324 (2003).

9. G. Altshuller, And Suddenly the Inventor Appeared TRIZ, the Theory of Inventive Problem Solving, 2nd ed., (1996) 